\title{
Dispersion of passive particles by a quasi-two-dimensional turbulent flow
}

\author{
I. M. Sokolov \\ Theoretical Polymer Physics, University of Freiburg, Hermann-Herder-Strasse 3, D-79104 Freiburg im Breisgau, Germany \\ R. Reigada \\ Department de Química Física, Universitat de Barcelona, Diagonal 647, Barcelona 08028, Spain \\ and Department of Chemistry and Biochemistry 0340, University of California, San Diego, La Jolla, California $92093-0340$
}

(Received 5 October 1998)

\begin{abstract}
Using the experimental data of Paret and Tabeling [Phys. Rev. Lett. 79, 4162 (1997)] we consider in detail the dispersion of particle pairs by a two-dimensional turbulent flow and its relation to the kinematic properties of the velocity field. We show that the mean square separation of a pair of particles is governed by rather rare, extreme events and that the majority of initially close pairs are not dispersed by the flow. Another manifestation of the same effect is the fact that the dispersion of an initially dense cluster is not the result of homogeneously spreading the particles within the whole system. Instead it proceeds through a splitting into smaller but also dense clusters. The statistical nature of this effect is discussed. [S1063-651X(99)09804-9]
\end{abstract}

PACS number(s): 47.27.Qb, 05.40.-a

Understanding the dispersion of particles in a turbulent velocity field is both of practical importance and of much theoretical interest. It is of fundamental importance for the correct modeling of turbulent diffusion and mixing properties of flows, a basic issue in environmental studies. A great deal of experimental insight has been gained following the pioneering work of Richardson (1926), Ref. [1], but a full understanding of the problem is still lacking (see Ref. [2] for a review).

Experimental studies of particle dispersion by turbulent flows are always complicated by molecular diffusivity or buoyancy effects. Moreover, it is difficult to connect directly the properties of dispersion to the Lagrangian statistics of the velocity field since the particles' positions and the velocities are usually not measured simultaneously in one experiment, for the same flow realization. However, applying the digital particle-image velocimetry method to model flows in twodimensional cells one can obtain velocity fields $\mathbf{v}(\mathbf{r}, t)$ with fine spatial and temporal resolution. These can then be used both for the analysis of Eulerian characteristics of the velocity field and for gaining arbitrary Lagrangian information by integrating the equations of motion of "fictive" particles (fluid elements)

$$
\frac{d \mathbf{r}}{d t}=\mathbf{V}(\mathbf{r}, t)
$$

starting from a given initial point $\mathbf{r}\left(t_{0}\right)$ at a given instant of time $t_{0}$. This "semiexperimental" fictive-particle approach to the transport processes under turbulence, pursued earlier in Refs. [3-5], may be extremely fruitful.

In this paper we focus on the properties of particle pair and cluster dispersion by a two-dimensional turbulent flow generated in a shallow cell under electromagnetic forcing and density stratification. The most attention will be paid to the connection between the dispersion and the relative velocity correlation function, a quantity which is fundamental for the theoretical understanding of dispersion, and which is hardly accessible otherwise. The experimental data were provided by Paret and Tabeling, and are the same as that used in
Refs. [3] and [5]. The details of the experimental setup, data acquisition, and preprocessing procedures can be found in the same publications. The velocity field, in a cell of approximate dimensions $16 \mathrm{~cm} \times 16 \mathrm{~cm}$, is digitalized on a 64 $\times 64$ square grid, corresponding to a grid constant of $a$ $=0.25 \mathrm{~cm}$. This gives an array of 4096 velocity vectors $\mathbf{V}_{i, j}(t)$ per time step of $\Delta t=0.04 \mathrm{sec}$, from $t=0$ until $t_{\max }$ $=60 \mathrm{sec}$. The fine time-discretization allows use of $\Delta t$ as the integration step. In space, the value of the velocity $\mathbf{V}(\mathbf{r})$ $=\left(\mathbf{V}^{x}(\mathbf{r}), \mathbf{V}^{y}(\mathbf{r})\right)$ at the point $\mathbf{r}=(x, y)$ is interpolated using the bilinear form

$$
\begin{aligned}
V^{\alpha}(\mathbf{r})= & (1-\xi)(1-\eta) V_{p, q}^{\alpha}+(1-\xi) \eta V_{p, q+1}^{\alpha} \\
& +\xi(1-\eta) V_{p+1, q}^{\alpha}+\xi \eta V_{p+1, q+1}^{\alpha}
\end{aligned}
$$

where $\alpha$ denotes the velocity's Cartesian component, $p$ $=[x / a]$ and $q=[y / a]$ give the coordinates of the grid's cell in which the point $\mathbf{r}$ is situated, and the values of $\xi=\{x / a\}$ and $\eta=\{y / a\}$ determine the relative position of a point within the cell. Here $[z]$ and $\{z\}$ denote the whole and fractional parts of $z$, respectively. This type of interpolation gives continuous coordinate-dependence for the velocity's spatial partial derivatives, and thus extrapolates reasonably well to small scales first-order differential forms such as rot $\mathbf{V}$ or the strain tensor. The interpolation allows to use a simple Euler algorithm for integrating Eq. (1) and to start integration from interparticle separations smaller that the grid constant in order to use the whole dynamic range of data.

As shown in Ref. [3], the flow (although twodimensional) shows the typical Kolmogorov-Obukhov $k^{-5 / 3}$ energy spectrum (within the $k$ interval of almost one order of magnitude in wave number). The possibility of the appearance of the Kolmogorov-Obukhov $k^{-5 / 3}$ scaling in twodimensional turbulent flow generated by inverse cascade is not surprising and was predicted in late 1960s in Refs. [6] and [7]. In Ref. [5] Richardson's law $\left\langle r^{2}(t)\right\rangle \propto t^{3}$ for the particle dispersion was observed within a time window of duration slightly smaller than one order of magnitude. The aim of 


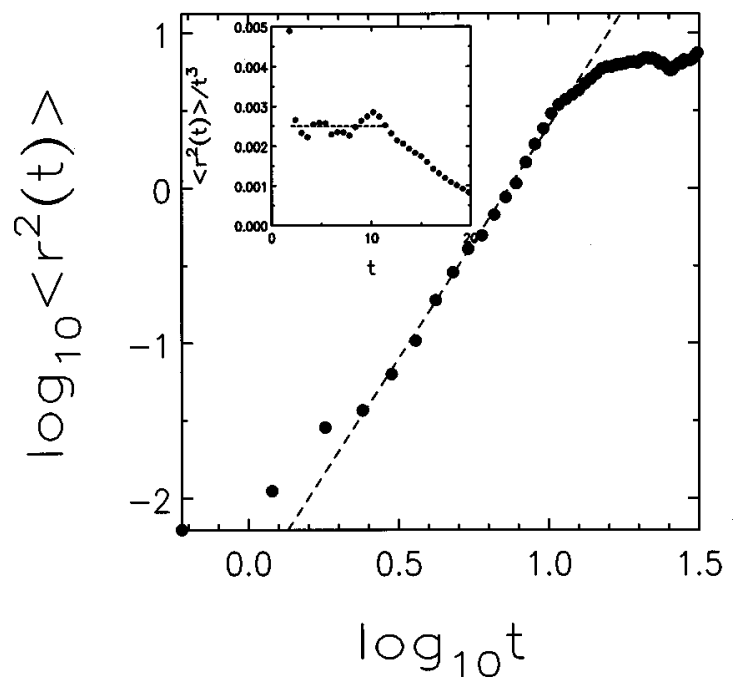

FIG. 1. Mean square displacement $\left\langle r^{2}(t)\right\rangle$ as a function of time shown on double logarithmic scales. The dashed line has the slope 3 . The inset shows the behavior of $\left\langle r^{2}(t)\right\rangle / t^{3}$; the horizontal line corresponds to the value of $R=0.0025$.

the present work is to connect the appearance of the Richardson's law with the correlation properties of the velocity field is space and time.

Starting from the set of close particles with initial separation fixed at $r_{0}=0.05 \mathrm{~cm}$ and initially placed within a square of size $1 \mathrm{~cm} \times 1 \mathrm{~cm}$ in the center of the system we integrate Eq. (1) for the particle pairs and average over $N=15000$ initial positions. The maximal integration time is chosen in such a way that the particles do not come into close vicinity of a boundary so as to avoid finite-size effects. The time dependence of $\left\langle r^{2}(t)\right\rangle$, within a time range from 2 to $10 \mathrm{sec}$ can be well fitted by the cubic form

$$
\left\langle r^{2}(t)\right\rangle \approx R t^{3},
$$

with the constant $R=(2.5 \pm 0.1) \times 10^{-3}$, as shown in Fig. 1, where the behavior of $\left\langle r^{2}(t)\right\rangle$ is plotted on double logarithmic scales. The value of $R$ is obtained as a mean value of $\left\langle r^{2}(t)\right\rangle / t^{3}$ for $2 \mathrm{sec}<t<10 \mathrm{sec}$ and the error bars denote the standard deviation of the mean. The inset shows the time dependence of $\left\langle r^{2}(t)\right\rangle / t^{3}$, which renders evidence of the existence of a plateau corresponding to Richardson's law and shows the value of $R$. The value of $R$ found here agrees with one that can be infered from Fig. 18 of Ref. [5] using another initial distribution of particles. The time range $2 \mathrm{sec}<t$ $<10 \mathrm{sec}$ will be referred to as Richardson's range. Our next task will be to relate this information to features of the kinematics of the velocity field.

The relative velocity $\mathbf{v}(r)$ of two particles depends on the mutual distance $r$ and its mean square value $\left\langle\mathbf{v}^{2}(r)\right\rangle$ is (in the stationary regime) a function of $r$ only [8,9]. Its radial part, responsible for the particle dispersion, behaves typically as

$$
v_{r}^{2}(r) \propto \begin{cases}A r^{2} & \text { for } r<\lambda \\ B r^{2 / 3} & \text { for } \lambda<r<L \\ \text { const } & \text { for } r>L,\end{cases}
$$

where $\lambda$ is the characteristic viscous scale and $L$ is the upper dimension of the Kolmogorov range. The coefficient $B$ is

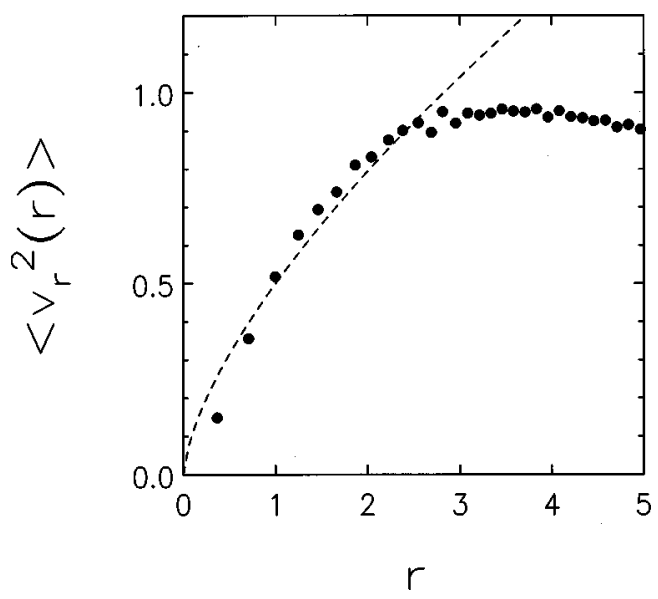

FIG. 2. Mean square radial component of relative velocity of a flow $\left\langle v_{r}^{2}(r)\right\rangle$ (in $\mathrm{cm} / \mathrm{sec}$ ), as a function of a distance (in $\mathrm{cm}$ ). The dashed line represents the behavior $v(r)=0.26 r^{2 / 3}$ used in further estimates.

connected with the energy dissipation rate in the flow [9]. The behavior of $v_{r}^{2}(r)$ obtained from the experimental data is shown in Fig. 2. The characteristic value of $L$ (viewed as a crossover from the power law to a plateau region) can be estimated as $L \approx 3 \mathrm{~cm}$. A rough estimate for the lower boundary, based on a $\log -\log$ presentation of $v_{r}^{2}(r)$, gives $\lambda \approx 0.5 \mathrm{~cm}$. This value is of the same order of magnitude as the characteristic discretization length of the system, $a$ $=0.25 \mathrm{~cm}$, and is thus somewhat ambiguous. The coefficient $B$ obtained by a least-square-fit to the data in the range $0.5 \mathrm{~cm}<r<3 \mathrm{~cm}$ is $B \approx(0.26 \pm 0.02) \mathrm{cm}^{4 / 3} / \mathrm{sec}^{2}$.

The value of the constant $R$ is connected to the behavior of $v(r)$ as a function of $r$ and to the temporal correlations of the relative velocities, see Refs. [10] and [11]. Note that the $t^{3}$ law stems essentially from pairs of particles moving "ballistically," i.e., in such a way that the direction of the velocity is fixed, so that the relative distance is increasing. To estimate the mean square separation one can start from integrating the ballistic equation of motion for the relative distance, $d r / d t=\nu_{\text {sep }}(r)$, and take the separation velocity to be of the order of rms relative velocity at the distance $r$, $\nu_{\text {sep }}(r) \cong\left[\nu_{r}^{2}(r)\right]^{1 / 2}$, as in Ref. [9]. For the mean square separation one then gets

$$
r^{2}=A(\sqrt{B} t)^{3}
$$

with the numerical coefficient $A$ being of the order of unity (but dependent on the exact assumptions done). Equation (5) typically overestimates $R$ by 1.5-2 orders of magnitude compared to its measured value (this is too strong a discrepancy even for a simple scaling estimate). This means that only a small part of the pair population really separates in a ballistic way and thus contributes considerably to the overall dispersion, i.e., it indicates that the dispersion in a two-dimensional velocity field is governed not by typical but by rare, extreme events (possibly Lévy-enhanced, as proposed in Ref. [11]).

The associated population can be estimated by calculating the direction correlation function of the relative motion $\Psi(t)=\left\langle\mathbf{e}\left(t_{0}\right) \mathbf{e}\left(t_{0}+t\right)\right\rangle$, where the average is taken over the pairs and over the times $t_{0}$. Here $\mathbf{e}(t)$ is a unit vector in the 


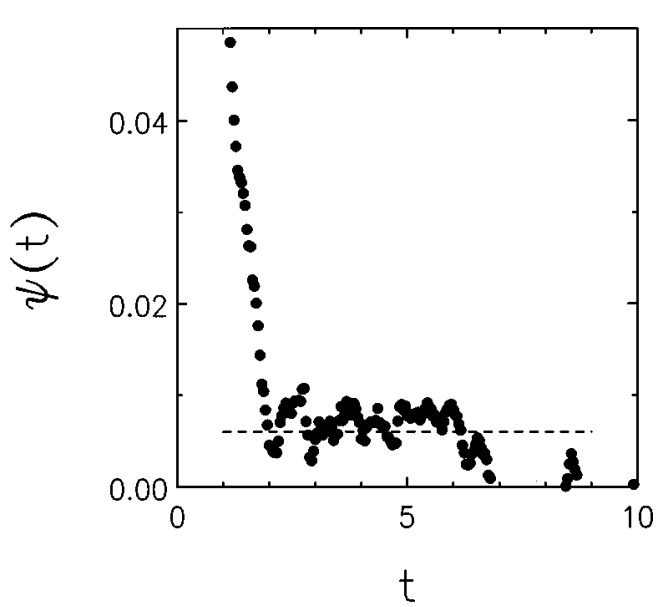

FIG. 3. Correlation function of the direction of relative motion, $\Psi(t)=\left\langle\mathbf{e}\left(t_{0}\right) \mathbf{e}\left(t_{0}+t\right)\right\rangle$, as a function of time (sec). Note the plateau in $\Psi(t)$ for $2 \mathrm{sec}<t<7 \mathrm{sec}$. The height of the plateau (obtained as the function's mean value in this time interval) is approximately 0.006 and is shown as a dashed line.

direction of the relative velocity of a pair of particles at time $t, \mathbf{e}(t)=\mathbf{v}(t) /|\mathbf{v}(t)|$, and $\mathbf{v}(t)$ is a relative velocity of a pair at time $t$. The function $\Psi(t)$ is presented in Fig. 3 and shows rather unexpected behavior: at short times, $t<2 \mathrm{sec}$ it decays exponentially, but then it stagnates and reaches a plateau region lasting almost throughout the whole Richardson's range, until $t=7 \mathrm{sec}$. After this it starts to oscillate near zero, these oscillations probably have purely statistical character. The height of the plateau obtained as a mean value of $\Psi$ in the interval $2 \mathrm{sec}<t<7 \mathrm{sec}$ is approximately $\bar{\Psi} \approx 6 \times 10^{-3}$.

The important role played by $\Psi(t)$ is rendered clear by turning to a Drude-like kinematic description of dispersion [11], based on a Lévy-walk-picture of Ref. [12]. In Ref. [11] the dispersion process was considered to be due to a laminar ballistic motion interrupted by distinct scattering events. After such an event the direction of the relative velocity is chosen at random. In this model the function $\Psi(t)$ is exactly the probability that no scattering has taken place during the time $t$, and thus the value of $\bar{\Psi}$ is a good estimate of the effective fraction of particles undergoing ballistic separation. Note that the simple weighing of the value of $r^{2}(t)$, given by Eq. (4), with the factor $\bar{\Psi}$ gives an estimate for the proportionality factor $R$ in Eq. (3), which has the correct order of magnitude. This estimate shows that only the pairs that undergo ballistic separation contribute considerably to the relative dispersion. The number of such pairs, keeping constant the average direction of the relative velocity for considerable times, is small. The pairs whose relative velocity has changed many times, contribute much less to the overall dispersion; the particles in such pairs stay closer together during considerable time. Thus, the Richardson's behavior in the case considered stems not from the typical separation in a pair of particles, but from the extremely and ballistically separated ones.

To prove this assumption we calculated the distribution of the interparticle distances at different times and mostly concentrated on the low-order integral characteristics of this distribution. Thus, in addition to the mean-square distance $\sigma^{2}(t)=\left\langle r^{2}(t)\right\rangle$ we calculated the mean absolute distance

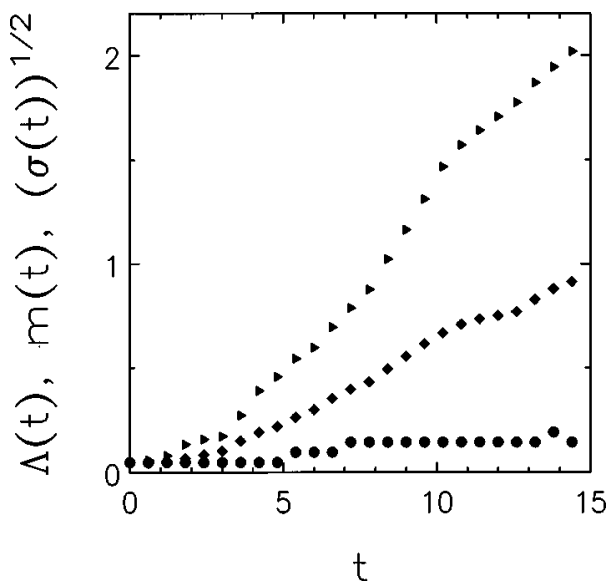

FIG. 4. Time dependence of the median $\Lambda(t)$ (circles), mean value $m(t)$ (diamonds), and the variance $[\sigma(t)]^{1 / 2}$ (triangles) of the distribution of the interparticle distances as functions of time. See text for details.

$m(t)=\langle r(t)\rangle$ and the median $\Lambda(t)$, defined as the distance such that exactly $N / 2$ of the pairs have the separation $r$ $\leqslant \Lambda$. The time evolution of $\sigma(t)=\left\langle r^{2}(t)\right\rangle^{1 / 2}, m(t)$, and $\Lambda(t)$ for the times within the Richardson's range is shown in Fig. 4. The results show that the relative first moment $z$ $=m(t) / \sigma(t)$ does not strongly change during the Richardson's range and eventually reaches the value of approximately 0.5 . On the other hand, the relative value of the median, $l$ $=\Lambda(t) / \sigma(t)$, at the end of the Richardson's range is approximately 0.075 . We compare these values to the ones following from the Richardson's diffusion approximation [1], describing the process by the equation for the distance neighbor function,

$$
\frac{\partial p(\mathbf{r}, t)}{\partial t}=\frac{\partial}{\partial r_{i}}\left(K(r) \frac{\partial p(\mathbf{r}, t)}{\partial r_{i}}\right)
$$

with the effective turbulent diffusivity $K(r)=\alpha r^{4 / 3}$. The rotation-symmetric solution of this equation in $2 \mathrm{D}$ reads

$$
p(r, t)=\frac{243}{64}(\alpha t)^{-3} \exp \left(-\frac{9 r^{2 / 3}}{4 \alpha t}\right) .
$$

From Eq. (7) the time-independent, universal values of $z$ and $l$ follow. That is, $z=7 \sqrt{15 \pi} / 64 \approx 0.751$ and $l$ is approximately 0.565 . The value of $l$ is equal to $l=\zeta^{3 / 2} / 2 \sqrt{15}$, with $\zeta$ is a root of the transcendent equation $e^{\zeta}=2+2 \zeta+\zeta^{2}$. We see that both values are larger than those obtained through simulations. Especially drastic is the discrepancy in the values of $l$; the value from diffusion approximation is almost 2 orders of magnitude larger than the "experimental" one. This means that the typical pair is hardly dispersed by the flow, and that particles initially close together stay clustered during considerable times. This finding parallels the picture of dispersion of two close particles following from the simulations of Ref. [13].

Let us confront these findings with the behavior of single particles. The fractal non-Brownian trajectories of particles were found in many different types of fluid motion, e.g., in chaotic flows [14], in motion of particles driven by capillary waves [15], and in two-dimensional turbulent flows, both 


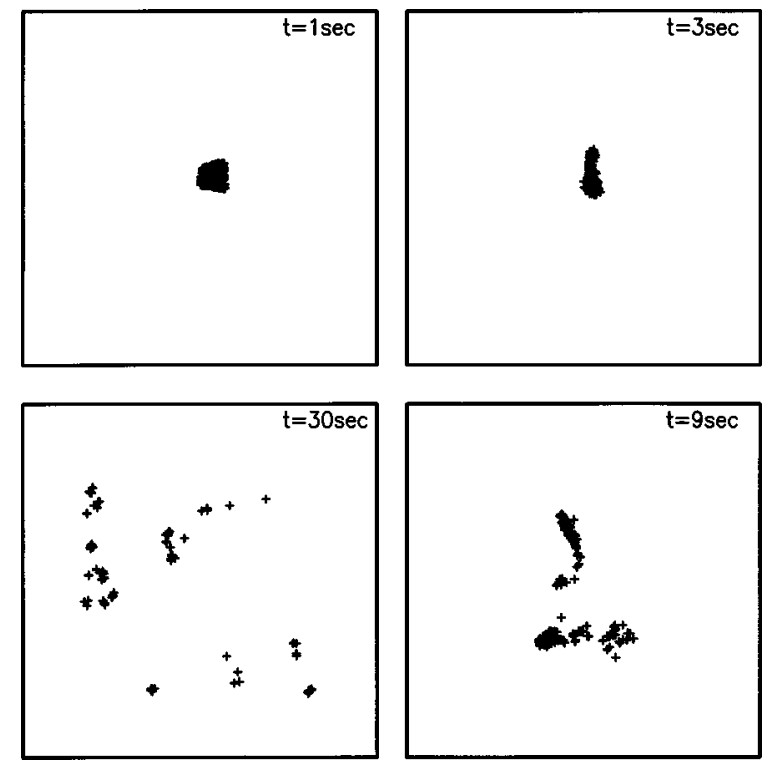

FIG. 5. Snapshots of the positions of 400 particles (shown as crosses) starting a $t=0$ in a square of a side $1 \mathrm{~cm}$ in the middle of the system at $t=1,3,9$, and $30 \mathrm{sec}$. Note that particles' dispersion results from stretching and folding the initial region. Note also that even at $t=30$ strong clustering is present.

decaying and stationary [5]. In all these cases the typical behavior of tracer particles can be considered as a sequence of periods of fast, directed motion (flights) interrupted by periods of slow, erratic motion (quoted as traps in Ref. [14]). When we turn to the consideration of pairs of particles, we find that most of the initially close pairs stay close to each other during considerable times, performing similar motions both during the flight and the trapping periods, and following close trajectories in space. On the other hand, this means that the large separations giving rise to the overall dispersion (i.e., flights in the relative motion of particles) are much more rare than when assuming the particles to move independently from each other. On the other hand these events (although rare) are the ones contributing considerably to Richardson's dispersion.

We illustrate the cluster nature of the particles' dispersion by presenting the result of calculations in which we simultaneously trace $N=400$ particles, initially placed within a $1 \mathrm{~cm} \times 1 \mathrm{~cm}$ square. The size of the region is laying within the inertial range, so that the region is inevitably dispersed. We show snapshots of the particles' positions at $t=1,3,9$, and $30 \mathrm{sec}$ in Fig. 5. We see that particles' dispersion results from stretching and folding the initial region. On the other hand, even at very long times the particles stay clustered in few dense "clouds," and only few of them are present as solitary ones. This cluster nature of turbulent dispersion was already clear to Richardson (see Figs. 1-5 of Ref. [1]), but was rarely stressed after him. Thus, the mean square distance between the particles is mostly governed by the characteris- tic distance between the clusters; the characteristic dimension of the clusters is only slightly larger than $\lambda$. The existence of clusters (clouds) is a very important feature of turbulent mixing in 2D and must be of extreme importance when considering, for example, the pollutants' spreading in the atmosphere. This clustering leads to a distribution of interparticle distances showing the pronounced peak at small distances. This finding is parallel to the results of the Lévywalks based Drude-approach of Ref. [11]. Following a "realtime" development of the particles' position on a screen as a sort of movie we find that the dispersion mechanism corresponds in general to stretching and detachment of clusters, but that situations in which two clusters or individual particles merge together and travel for a while as a compact cloud also occure.

The clustering phenomenon shows the week mixing ability of a quasi-two-dimensional turbulent flow. This finding is parallel to the results of experiments of Ref. [16], where the measured spectra of passive scalars stirred by similar turbulent flows could be fitted only supposing the effective strain rates $\gamma_{\text {eff }}$ along the Lagrangian trajectories which are 3 orders of magnitude smaller than the principal strain rate averaged over the system's volume. Of course, some reservations must be made when extrapolating our findings for quasi-twodimensional flows to purely two-dimensional and to threedimensional ones. The two-dimensional velocity fields obtained as projections of instantaneous velocity fields in a shallow cell on a horizontal plane are not absolutely divergence-free, due to possible vertical motions. The characteristic values of divergence are small, being a $10^{-3}$ of $v_{0} / L$. We calculated the divergence charts for the same times as when the positions of the particles were calculated, and plotted them together with the particles' positions. The clusters are not associated with the regions in the flow with negative instantaneous divergence. On the other hand, the nonzero divergences, although small but persisting over longer times, could nevertheless be manifested on the background of stochastic mixing, thus interferring with turbulent stirring.

To conclude, we summarize our findings. Using the experimental data for turbulent velocity fields in a shallow cell, we consider the dispersion of pairs and clusters of particles by such quasi-two-dimensional turbulent flow. We show that the mean square separation of a pair of particles in such flow is connected with rare and extreme events. The connection of these findings with the correlation properties of the velocity field in space and time is discussed.

We are extremely grateful to J. Paret and P. Tabeling for providing the experimental velocity fields. We are thankful to A. Blumen, J. Klafter, K. Lindenberg, F. Sagués, and J. M. Sancho for fruitful discussions. The support of the DFG (SFB 428) and of an "ACCIONES INTEGRADAS"DAAD grant are gratefully acknowledged.
[1] L. F. Richardson, Proc. R. Soc. London 110, 709 (1926).

[2] A. Tsinober, in Lévy Flights and Related Topics in Physics, edited by M. F. Shlesinger, G. M. Zaslavsky, and U. Frisch (Springer-Verlag, Berlin, 1995).
[3] J. Paret and P. Tabeling, Phys. Rev. Lett. 79, 4162 (1997).

[4] J. Paret, D. Marteau, O. Pareau, and P. Tabeling, Phys. Fluids 9, 3102 (1997).

[5] P. Tabeling, A. E. Hansen, and J. Paret, in Chaos, Kinetics and 
Nonlinear Dynamics in Fluids and Plasmas, edited by G. M. Zaslavsky and S. Benkadda (Springer, Berlin, in press).

[6] R. H. Kraichnan, Phys. Fluids 10, 1417 (1967).

[7] C. E. Leith, Phys. Fluids 11, 671 (1968).

[8] A. N. Kolmogorov, Dokl. Akad. Nauk SSSR 30, 301 (1941).

[9] L. D. Landau and E. M. Lifshitz, Fluid Mechanics, 2nd ed. (Pergamon, Oxford, 1987).

[10] G. K. Batchelor, Q. J. R. Meteorol. Soc. 76, 133 (1950).

[11] I. M. Sokolov, A. Blumen, and J. Klafter (unpublished).
[12] M. F. Shlesinger, B. West, and J. Klafter, Phys. Rev. Lett. 58, 1100 (1987).

[13] S. Grossmann and C. Wiele, Z. Phys. B 103, 469 (1997).

[14] T. H. Solomon, E. R. Weels, and H. L. Swinney, Physica D 76, 70 (1994).

[15] A. E. Hansen, E. Schröder, P. Alstrøm, J. S. Andersen, and M. T. Levinsen, Phys. Rev. Lett. 79, 1845 (1997).

[16] B. S. Williams, D. Marteau, and J. P. Gollub, Phys. Fluids 9, 2061 (1997). 